\title{
Studies on In situ Magnetic Alignment of Bonded Anisotropic Nd-Fe-B Alloy Powders
}

\author{
I. C. Nlebedim ${ }^{1}$, Huseyin Ucar ${ }^{2}$, Christine B. Hatter ${ }^{2}$, R. W. McCallum ${ }^{1}$, Scott K. McCall ${ }^{3}$, M. J. Kramer ${ }^{1}$ \\ and M. Parans Paranthaman ${ }^{2}$ \\ ${ }^{1}$ Ames Laboratory, Ames, Iowa 50011, USA \\ ${ }^{2}$ Oak Ridge National Laboratory, Oak Ridge, Tennessee 37831, USA \\ ${ }^{3}$ Lawrence Livermore National Laboratory, Livermore, California 94550, USA
}

\begin{abstract}
Considerations for achieving high degree of alignment in polymer bonded permanent magnets are presented via the results of a study on in situ magnetic alignment of anisotropic Nd-Fe-B magnet powders. Contributions from effect of the alignment temperature, alignment magnetic field and the properties of the polymer on the hard magnetic properties of the bonded magnet were considered. The thermo-rheological properties of the polymer and the response of the magnet powders to the applied magnetic field indicate that hard magnetic properties were optimized at an alignment temperature just above the melting temperature of the EVA co-polymer. This agrees with an observed correlation between the change in magnetization due to improved magnetic alignment of the anisotropic powders and the change in viscosity of the binder. Manufacturing cost can be minimized by identifying optimum alignment temperatures and magnetic field strengths.
\end{abstract}

\section{INTRODUCTION}

The growth in compact electronic devices has resulted in the need to develop net-shape high performance permanent magnets with minimal post fabrication machining. Machining of sintered Nd-Fe-B magnets adds to manufacturing costs and results in significant waste of materials in the form of grinding or cutting swarfs [1]. Bonded magnets can easily be made into desired shapes with minimal or no post manufacturing machining. As a result, they are suitable for applications in which post-manufacturing processing limits the use of sintered magnets and are therefore well suited for advanced manufacturing technologies. They are typically manufactured by mixing magnet powders with a binder of choice, pouring the mixture into a mold and subjecting it to a hardening (curing) process, as schematically shown in Fig. 1 [2-5].

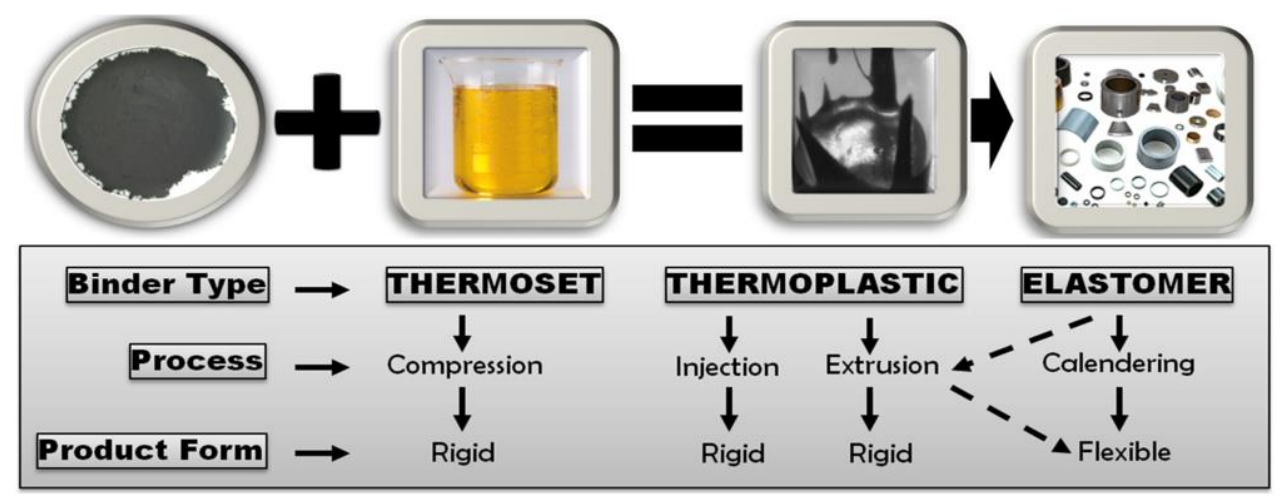

Fig.1. Schematic of bonded magnet manufacturing in which a magnet powder is mixed with a binder and cured to obtain desired shapes (top) Bonded magnet manufacturing processes allow the use of different binder types to produce both rigid and flexible bonded magnets (bottom).

Fig. 1 also shows that bonded magnets can be made both in rigid and flexible forms; making them suitable for many applications [6,7]. The dilution of the magnetic properties of the magnet powders in non-magnetic media, such as polymer binders, results in low energy products $(\mathrm{BH})_{\max }$. Commercially available bonded Nd-Fe-B magnets typically have (BH) $\max _{\max }$ of 10-12 MGOe. The achievable $(\mathrm{BH})_{\max }$ depends on the magnetic properties of the magnet powders and the loading fraction in the binder [4], 
assuming that the manufacturing processes do not deteriorate the properties of the powder. The loading fraction, in turn, depends on the molding process selected (Fig. 1) [8]. In some applications, however, manufacturability, mechanical properties and the ability to withstand corrosive environments may be more limiting than high $(\mathrm{BH})_{\max }$. The binders can also be used to improve mechanical properties and corrosion resistance [9]. For applications in which the obtainable magnetic properties are sufficient, bonded magnets can help address criticality in rare-earth materials for permanent magnets by minimizing post-manufacturing processing wastes while using smaller quantities of magnetic materials, compared to sintered magnets.

Unlike isotropic magnet powders, anisotropic powders can be magnetically aligned during the manufacturing of bonded magnets, which provides an avenue for enhancing $(\mathrm{BH})_{\max }$ by increasing the remanent magnetization. In addition, specific alignment configurations can be used to control the flux path in a magnetic circuit using anisotropic bonded magnets. Although the thermo-rheological properties of the polymer binders typically used in the manufacturing of bonded magnets may limit the maximum operating temperatures, it is worth noting that some polymers have operating temperatures higher than the maximum operating temperatures of many of the $\mathrm{Nd}-\mathrm{Fe}-\mathrm{B}$ magnet grades [10-12]. Enhancing the hard magnetic properties of bonded magnets via the use of anisotropic powders requires achieving a high degree of powder alignment within the binder. In order to determine the effect of processing conditions on the hard magnetic properties, we performed in situ magnetic alignment and magnetic properties measurement studies on anisotropic Nd-Fe-B magnet powders in a polymer binder; considering the effects of alignment temperature, magnetic field strength and the properties of the binder.

\section{EXPERIMENTAL APPROACH}

Polymer bonded magnets were produced by extrusion using commercial anisotropic magnet powder (Magnequench MQA) and ethylene-vinyl acetate (EVA) copolymer. The initial magnetic properties of the MQA and the thermal transitions of the EVA were determined with a SQUID magnetometer and differential scanning calorimeter (DSC), respectively. The as-received MQA powder has intrinsic coercivity, $\mathrm{H}_{\mathrm{ci}}=12 \mathrm{kOe}$ and remanence, $\mathrm{M}_{\mathrm{r}}=12.9 \mathrm{kG}$. The powder was rated for a $(\mathrm{BH})_{\max }$ of $38 \mathrm{MGOe}$. DSC measurements were performed at 10 degrees/min in a He atmosphere. The second cycle of the DSC measurement, without the contributions from the initial processing of the polymer, was used to determine the properties of the EVA [13]. $50 \mathrm{vol} \%$ of each of MQA and EVA were fed into a twin screw mixer, heated to $125{ }^{\circ} \mathrm{C}$ while being mixed with rotating screws in the mixer at $80 \mathrm{rpm}$ for 10 minutes, and afterwards extruded. Unaligned bonded magnet samples from the extruded materials were inserted into 3 $\mathrm{mm}$ diameter quartz test tubes, and aligned in the SQUID magnetometer. Magnetic hysteresis loops at $300 \mathrm{~K}$ of the as-made bonded magnets were measured for each sample prior to in situ alignment in the SQUID magnetometer. Samples were then heated in a magnetic field, $\mathrm{H}_{\mathrm{i}}$, from $300 \mathrm{~K}$ to temperature, $\mathrm{T}_{\mathrm{i}}$, soaked for 15 minutes with magnetic field still being applied, and then cooled to $300 \mathrm{~K}$, where magnetic hysteresis loops were measured again. For each alignment magnetic field strength, $H_{i}=10,20,30,40$ and $50 \mathrm{kOe}$, samples were heated to alignment temperatures, $\mathrm{T}_{\mathrm{i}}=325 \mathrm{~K}, 350 \mathrm{~K}, 375 \mathrm{~K}$ and $400 \mathrm{~K}$ at 10 degrees/min. Magnetization as a function of temperature was measured as each sample was heated at constant $\mathrm{H}_{\mathrm{i}}$.

\section{RESULTS AND DISCUSSION}

The SEM micrograph in Fig. 2a shows a large particle size distribution of the as-received MQA powder. Fig. $2 \mathrm{~b}$ shows the magnetic hysteresis loops of the four unaligned bonded magnet samples (50 vol\% of MQA in EVA) used for the in situ alignment, after correcting for less than $1 \%$ variation in magnetization arising from the variation in size distribution of the as-received powder. The DSC plot in Fig. $2 \mathrm{c}$ shows the effect of the MQA magnet powder on the properties of the EVA polymer binder. Without the MQA magnet powder, the EVA shows an endothermic peak at $328 \mathrm{~K}$ with onset temperature at $\sim 317 \mathrm{~K}$ and end temperature at $\sim 336 \mathrm{~K}$; corresponding to the melting of the EVA polymer binder. With the MQA powder, only the onset of the melting temperature for the EVA is observed. The absence of a clearly defined peak 
in the EVA+MQA sample is likely due to a reaction of the EVA with the resin coating applied to the MQA during manufacturing [14]. Both plots have no crystallization peaks indicating that the polymers were completely crystallized before being used for magnetic alignment. The melting peak at $328 \mathrm{~K}$ in Fig. $2 \mathrm{c}$ agrees with the observation that viscosity is much higher at $325 \mathrm{~K}$, compared to other alignment temperatures used in this study. Viscosity $\approx 206080 \mathrm{~Pa} . \mathrm{s}, 36630 \mathrm{~Pa} . \mathrm{s}, 10400 \mathrm{~Pa}$.s and $3320 \mathrm{~Pa}$.s were obtained for the polymer binder at $325 \mathrm{~K}, 350 \mathrm{~K}, 375 \mathrm{~K}$ and $400 \mathrm{~K}$, respectively.


Fig.2. (a) Micrograph showing the size distribution of the MQA powder used (b) Magnetic hysteresis loops of the four bonded magnets used, before alignment (c) Effect of MQA on the properties of EVA binder.

A figure comparing representative hysteresis loops of the bonded magnets in the aligned (parallel and perpendicular measurements) and unaligned states is provided as supplementary information (Fig. S1). The magnetic hysteresis loops obtained at $300 \mathrm{~K}$, after alignment at different temperatures and magnetic field strengths are also provided as supplementary data in Fig. S2. To compare the effect of alignment at different conditions, the remanence-to-saturation magnetization ratio $\left(\mathrm{M}_{\mathrm{r}} / \mathrm{M}_{\mathrm{s}}\right)$ is defined as the degree of alignment. In Fig. 3a, $\mathrm{M}_{\mathrm{r}} / \mathrm{M}_{\mathrm{s}}$ is seen to increase with alignment magnetic field. At all temperatures, the maximum $\mathrm{M}_{\mathrm{r}} / \mathrm{M}_{\mathrm{s}}$ was at $\mathrm{H}=50 \mathrm{kOe} . \mathrm{M}_{\mathrm{r}} / \mathrm{M}_{\mathrm{s}}$ increased most significantly between the unaligned state and alignment at $\mathrm{H}=10 \mathrm{kOe}$. Above $\mathrm{H}=10 \mathrm{kOe}, \mathrm{M}_{\mathrm{r}} / \mathrm{M}_{\mathrm{s}}$ increased less significantly between the alignment fields, except at $325 \mathrm{~K}$. The increase in $\mathrm{M}_{\mathrm{r}} / \mathrm{M}_{\mathrm{s}}$ at $325 \mathrm{~K}$ is relatively significant up to $\mathrm{H}=30 \mathrm{kOe}$. The viscoelastic properties of EVA, including the elastic storage and loss moduli, contribute to the resistance of the magnet particles to being rotated into the field direction by the alignment field. Although the viscoelastic properties decrease rapidly above the glass transition temperature, they can still be significant in the temperature range of the present study [15-19]. As a result, the EVA matrix sufficiently limited the rotation of the anisotropic magnet particles by the applied magnetic field at $325 \mathrm{~K}$, compared to alignment at other temperatures. An increasing $\mathrm{M}_{\mathrm{r}} / \mathrm{M}_{\mathrm{s}}$ was obtained between $325 \mathrm{~K}$ and $375 \mathrm{~K}$ (Fig. $3 \mathrm{~b}$ ), corresponding to reducing viscoelastic effects as the viscosity of the binder decrease. At $400 \mathrm{~K}$, the decrease in $\mathrm{M}_{\mathrm{r}} / \mathrm{M}_{\mathrm{s}}$ compared to $375 \mathrm{~K}$ may be related to the effect of stronger magnetostatic interactions between the particles at very low viscosity.
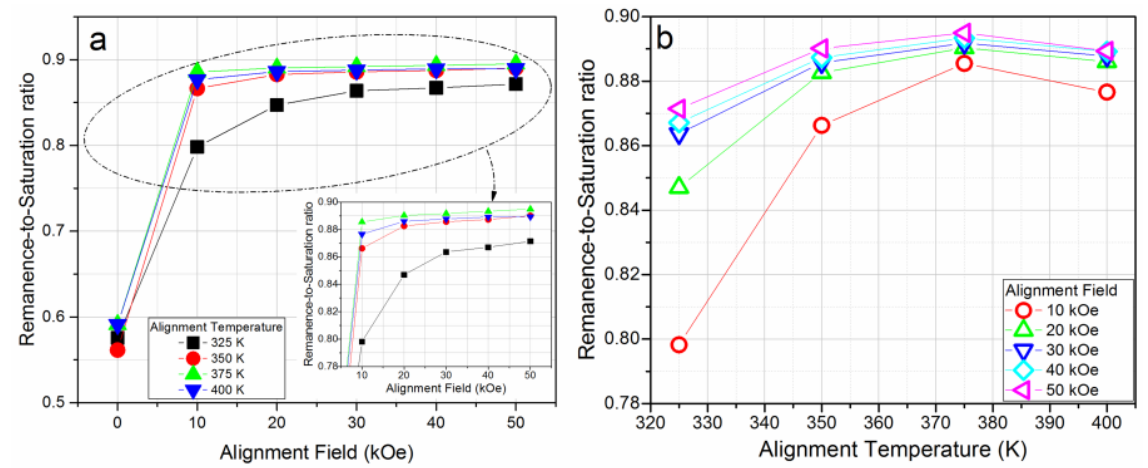

Fig.3. Dependence of remanence-to-saturation magnetization ratio on alignment (a) magnetic field (b) temperature 
Although the best degree of alignment was obtained at $50 \mathrm{kOe}$, the small deviation between the alignments at $20 \mathrm{kOe}$ and $50 \mathrm{kOe}$ suggests that the enhancement in properties may not justify the cost for generating magnetic fields above $20 \mathrm{kOe}$ for the alignment. Since magnetic field is proportional to applied current (Ampere's law) and power is proportional to the square of the applied current, then the power to achieve $\mathrm{H}=20 \mathrm{kOe}$ would be only $16 \%$ of the power to achieve $\mathrm{H}=50 \mathrm{kOe}$. This assumes generating both field strengths with the same type of equipment. In practice, the equipment cost for generating $50 \mathrm{kOe}$ would be significantly higher than that for generating $20 \mathrm{kOe}$. Therefore it may be more cost effective to use $\mathrm{H}=20 \mathrm{kOe}$ and increase the volume of the magnet powder in the binder to achieve comparable properties than requiring additional $84 \%$ of power supply for $\mathrm{H}=50 \mathrm{kOe}$. However, this should be balanced by the limitation to optimizing coercivity due to particle interactions at higher loading fractions, as will be further explained in discussing the effect on coercivity.

For all alignment temperatures shown in Fig. 4a, remanence increased with alignment magnetic field. Remanence increased more rapidly between the unaligned state and $10 \mathrm{kOe}$ alignment field. In previous studies $[20,21]$ in which alignment field up to $\mathrm{H}=15 \mathrm{kOe}$ were used, remanence was also reported to increase rapidly with the alignment magnetic field. The present study shows that, at higher magnetic field strengths, the increase in remanence is less rapid and tends to saturate. As shown in Fig. 4b, remanence increased with alignment temperature between $325 \mathrm{~K}$ and $350 \mathrm{~K}$. Although a slight decrease in remanence beyond $350 \mathrm{~K}$ can be seen, the values are comparable at higher alignment temperatures. For each temperature, the deviation of remanence from the mean value for the five alignment magnetic field strengths is shown in Fig. 4c. A lower deviation is desirable since it indicates that lower magnetic field strength can be used for alignment without significant change in remanence. This is because the resistance of the magnet powders to being rotated by the alignment magnetic field is minimized by the relatively much smaller viscosity of the polymer binder matrix at higher temperatures. Economically, this is also important because the energy cost for increasing the magnetic field strength would be significantly higher than that for increasing temperature. However, increasing temperature can affect the lifetime of the manufacturing tools, such as the molding dies.
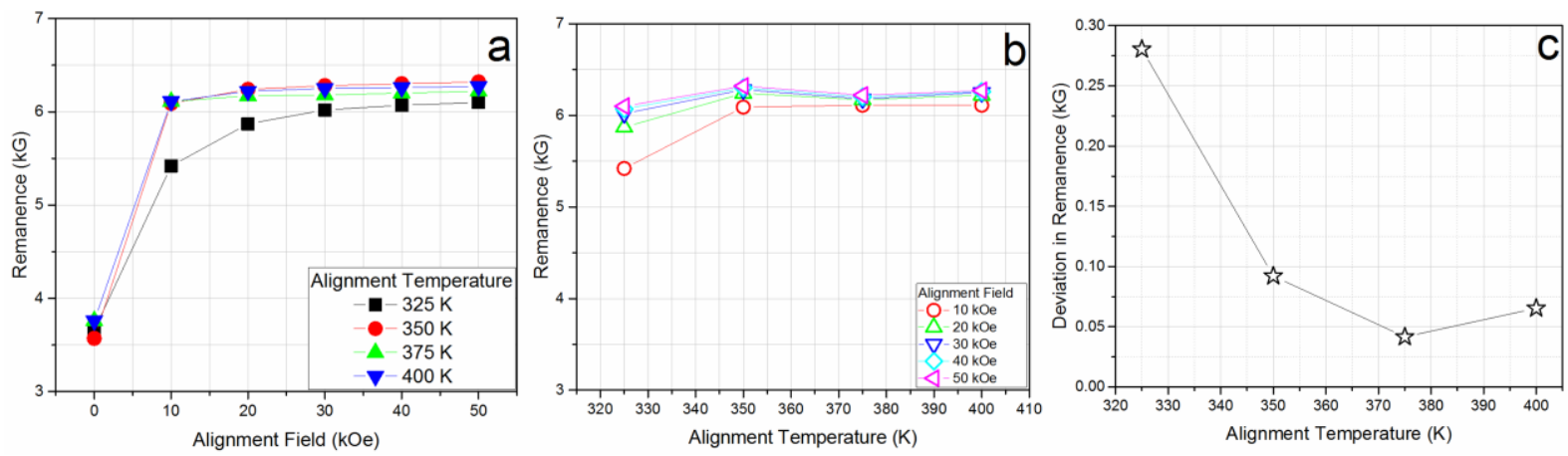

Fig.4. (a) Dependence of remanence on alignment magnetic field (b) Deviation of remanence obtained at 10, 20, 30,40 and $50 \mathrm{kOe}$ from mean value, as a function of alignment temperature (C) Dependence of remanence on alignment temneratures.

At each alignment temperature in Fig. 5a, coercivity increased between the unaligned states and alignment at $10 \mathrm{kOe}$ magnetic field. At $325 \mathrm{~K}$, coercivity increased continuously with alignment field but less significantly at higher field strengths. The values of coercivity obtained for alignment at $350 \mathrm{~K}$ remained comparable between $20 \mathrm{kOe}$ and $50 \mathrm{kOe}$. The same applies to $375 \mathrm{~K}$ and $400 \mathrm{~K}$, although a slight deviation seems to exist at higher alignment magnetic fields for both temperatures. The deviation of coercivity for the five alignment magnet fields is plotted against temperature in Fig. 5b. In practice, an alignment temperature with the least deviation in the coercivity values, corresponding to the alignment magnetic field strengths, is desirable for selecting lower magnetic field strength for alignment. In Fig. 5c, it can be seen that coercivity increased between $325 \mathrm{~K}$ and $350 \mathrm{~K}$ but decreased at higher temperatures. 
Two mechanisms (eq. 1 and eq. 2) affect the dependence of coercivity on the degree of alignment $[20,22,23]$. The Stoner-Wohlfarth mechanism (eq. 1) assumes a coherent rotation; which is applicable when the sizes of particles approach that of a single domain such that the effect of domain wall pining is negligible. $g(\theta)$ is the angular dependence of coercivity for magnetic field applied at an angle $\theta$ from the easy axis. The dependence of coercivity on the degree of alignment in the case of domain wall pinning is shown in eq. 2.

$$
\begin{array}{r}
g(\theta)=\frac{1}{\left(\sin ^{\frac{2}{3}} \theta+\cos ^{\frac{2}{3}} \theta\right)^{\frac{3}{2}}} \\
g(\theta)=\frac{1}{\cos \theta}
\end{array}
$$

For anisotropic powders in which there is a wide distribution of particle sizes (Fig. 2a), one would expect a deviation from the Stoner-Wohlfarth behavior on the dependence of coercivity on the degree of alignment. This is because, while very small particles may tend towards the single-domain particles requirement for the Stoner-Wohlfarth mechanism, larger particles will favor the domain wall pinning mechanism. Also depending on the alignment conditions, the effect of either of the mechanisms can dominate. A previous work in which the effect of alignment temperature was not considered reported a continuous increase in coercivity as alignment field increases up to a maximum of $\mathrm{H}=15 \mathrm{kOe}$, and concluded that that the Stoner-Wohlfarth mechanism was dominant [21]. That conclusion agrees with the observation in Fig. $5 \mathrm{c}$ for alignment between $325 \mathrm{~K}$ and $350 \mathrm{~K}$, irrespective of the alignment magnetic field. Nevertheless, beyond $350 \mathrm{~K}$, there is an obvious departure from that trend at all alignment magnetic fields; confirming the contribution from a different mechanism and the need to consider viscoelastic contribution from the polymer. For sintered magnets, it has been shown that the dependence of coercivity can be intermediate the Stoner-Wohlfarth (eq. 1) and Kondorsky (eq. 2) models [23]. A departure from the Kondorsky model has also been reported for hard magnetic films [24].
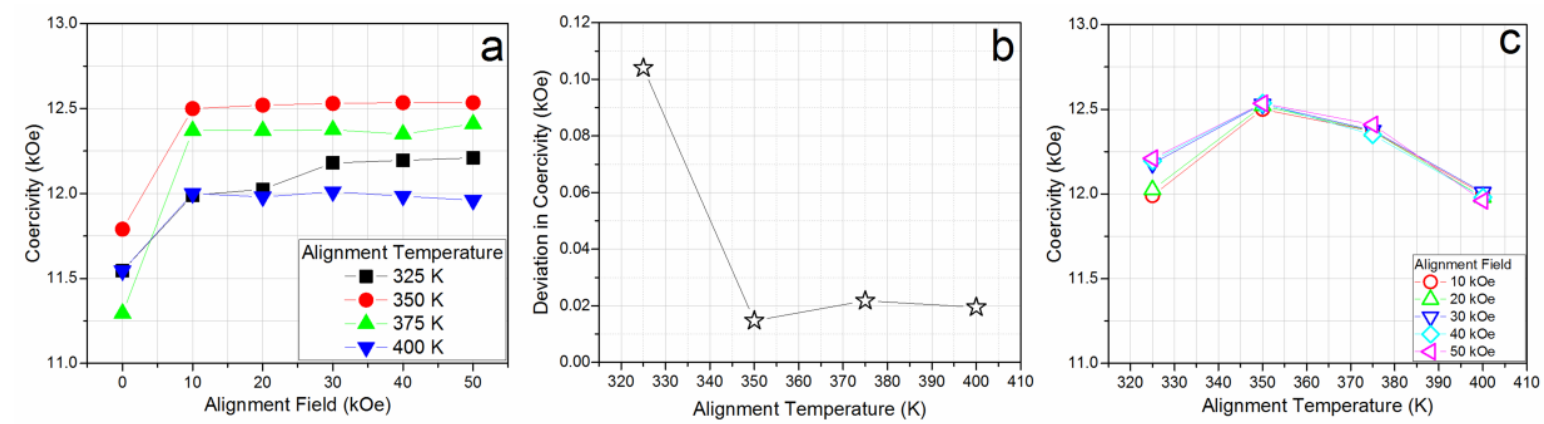

Fig.5. (a) Dependence of coercivity on alignment magnetic field (b) Deviation of coercivity obtained at 10, 20, 30, 40 and $50 \mathrm{kOe}$ from mean value, as a function of alignment temperature (c) Dependence of coercivity on alignment temperature.

A limitation of the Stoner-Wohlfarth mechanism is its assumption of non-interacting magnet particles. Compared to sintered magnets, bonded magnets in which small magnet particles are distributed in nonmagnetic binders are closer to meeting the non-interacting particles requirement. For polymer bonded magnets, the Stoner-Wohlfarth mechanism should dominate at lower temperatures because the elastic properties of the polymer would limit the movement of the particles, hence maximizing magnetic interaction length and limiting the interaction between the particles. Further increase in temperature will reduce viscosity, allow the particles to move, increase the magnetic interaction between the particles and also result in a departure from the Stoner-Wohlfarth mode, as seen in Fig. 4b. One would expect this effect to be stronger for magnet powders with large distributions of particle sizes. This dependence of the coercivity of bonded magnets on temperature and distribution of particle sizes can have significant practical consequence. This is because the strong magnetostatic interactions at the low viscous state of the 
polymer can allow the particles to move and result in irreversible changes in magnet particle distribution. Consequently, magnetic properties optimized based on the original distribution of the particles will be changed, hence any device performance dependent on such optimization of properties.

Fig. 6 shows the dependence of the $(\mathrm{BH})_{\max }$ on the alignment magnetic field and temperature. Since the MQA magnet powder was rated for a $(\mathrm{BH})_{\max }$ of 38 MGOe and because $(\mathrm{BH})_{\max }$ scales by the square of the loading fraction of magnet powder in the binder [25], a $(\mathrm{BH})_{\max }$ of $9.5 \mathrm{MGOe}$ is expected at optimum alignment conditions for the $50 \mathrm{vol} \%$ magnet powder in EVA. Also, since the $(\mathrm{BH})_{\max }$ is calculated from the second quadrant of the magnetization plot, the effects of alignment conditions on remanence and coercivity would be reflected on the effect on $(\mathrm{BH})_{\max }$. At each temperature in Fig. 6a, $(\mathrm{BH})_{\max }$ increased with alignment magnetic field. The trend is similar to that shown in Fig. 4a for remanence. In Fig. 6a and $6 \mathrm{~b}$, it is obvious that the highest values of $(\mathrm{BH})_{\max }$ was obtained at $350 \mathrm{~K}$. Above $350 \mathrm{~K}$, the difference in the $(\mathrm{BH})_{\max }$ obtained at all magnetic field strengths may justify using $\mathrm{H}=10 \mathrm{kOe}$ for alignment, rather than higher magnetic field strengths.
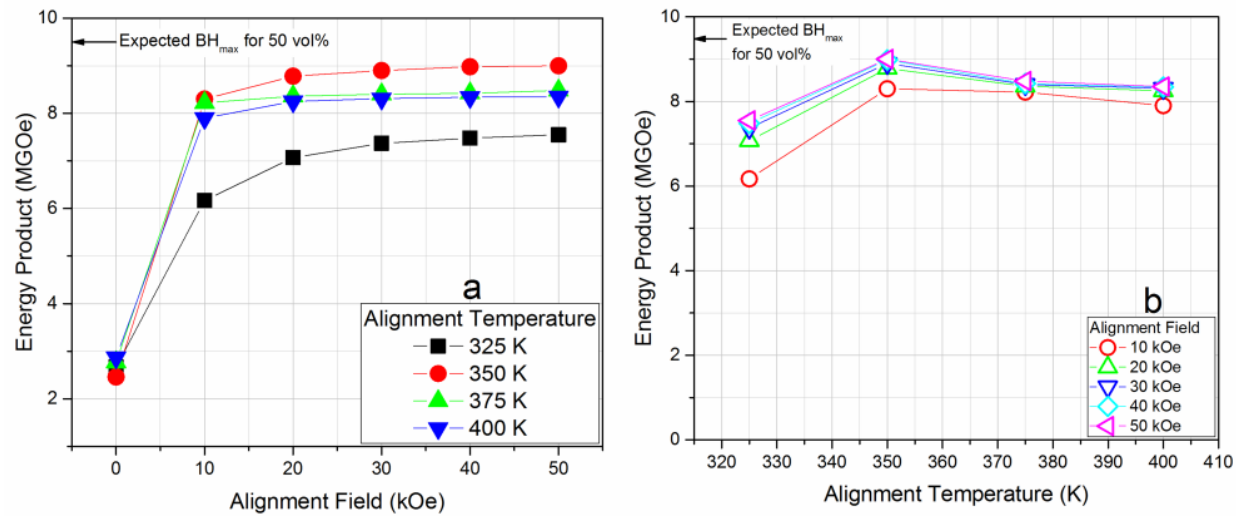

Fig.6. Effect of (a) alignment magnetic field and (b) alignment temperature on energy product.

Fig. 7 compares the effect of the alignment temperature on the melting characteristics of the polymer to the changes in the magnetization of the magnet powder. In Fig. 7a, the temperature after which magnetization started increasing is near the onset of the melting temperature in Fig. 7b. The temperature at which magnetization stopped increasing and the end of the melting event in the DSC plot, also correspond. This indicates that the highest change in magnetization is achieved when the binder transforms from being more elastic to being less viscous. Compared to other temperatures investigated in this work, $350 \mathrm{~K}$ is closest to the temperature corresponding to the end of the melting peak in the DSC at which the maximum magnetization was obtained. These observations suggest that the optimization of magnetic alignment in anisotropic bonded magnets depends both on the magnetic properties of the powders and the thermorheological changes in the polymer. Although not considered in the present study, the loading fraction of the powder in the binder can also play an important role.



Fig.7. Comparison of the magnetization (a) and differential scanning calorimetry (b) measurements, as a function of temperature. 


\section{CONCLUSION}

The results of a study on in situ alignment of anisotropic bonded magnets have been presented. Bonded magnet samples of MQA, an anisotropic Nd-Fe-B magnet powder and EVA copolymer, prepared by extrusion were aligned across an array of temperatures and applied magnetic fields to determine the optimum conditions for alignment. In addition to the applied magnetic field strength, hard magnetic properties also showed dependence on the interaction between the magnet particles due to the viscoelastic changes in the binder. For the EVA copolymer used, magnetic properties were enhanced at $350 \mathrm{~K}$ which corresponds to the measurement temperature closest to the end of the melting peak observed in differential scanning calorimetry. At lower magnetic field strengths, the Stoner-Wohlfarth mechanism dominated the contribution to coercivity while the domain wall pinning mechanism dominated at higher fields.

\section{ACKNOWLEDGMENT}

This work was supported by the Critical Materials Institute, an Energy Innovation Hub funded by the U.S. Department of Energy, Office of Energy Efficiency and Renewable Energy, Advanced Manufacturing Office. Work at Ames Laboratory, operated by Iowa State University, was performed under Contract No. DE-AC02-07CH11358. Work done at LLNL was prepared under Contract DE-AC52-07NA27344. Authors wish to acknowledge Jim Herchenroeder of Molycorp Magnequench for providing the MQA magnet powders used for this work.

\section{Notice of Copyright}

This manuscript has been authored by UT-Battelle, LLC under Contract No. DE-AC05-00OR22725 with the U.S. Department of Energy. The United States Government retains and the publisher, by accepting the article for publication, acknowledges that the United States Government retains a non-exclusive, paid-up, irrevocable, world-wide license to publish or reproduce the published form of this manuscript, or allow others to do so, for United States Government purposes. The Department of Energy will provide public access to these results of federally sponsored research in accordance with the DOE Public Access Plan (http://energy.gov/downloads/doe-public-access-plan).

\section{REFERENCES}

[1] T. Xu, H. Peng, Formation cause, composition analysis and comprehensive utilization of rare earth solid wastes, J. Rare Earths. 27 (2009) 1096-1102.

[2] J. Ormerod, S. Constantinides, Bonded permanent magnets: Current status and future opportunities (invited), J. Appl. Phys. 81 (1997) 4816.

[3] M. Gronefeld, Review on Bonded Magnets, in: G.C. Hadjipanayis (Ed.), Bond. Magnets, Kluwer Academic Publishers, Netherlands, 2003: pp. 1-2.

[4] B.. Ma, J.. Herchenroeder, B. Smith, M. Suda, D.. Brown, Z. Chen, Recent development in bonded NdFeB magnets, J. Magn. Magn. Mater. 239 (2002) 418-423.

[5] K. Chow, Bonded Magnet, MAGTECH Ind. CO. (n.d.).

[6] J.J. Croat, Current status and future outlook for bonded neodymium permanent magnets (invited), J. Appl. Phys. 81 (1997) 4804.

[7] Muljadi, P. Sardjono, Suprapedi, Preparation and Characterization of 5wt.\% Epoxy Resin Bonded Magnet NdFeB for Micro Generator Application, Energy Procedia. 68 (2015) 282-287.

[8] D. Zhang, Z. Ren, C.-S. Koh, Optimal Design of Powder-Aligning and Magnetizing Fixtures for an Anisotropic-Bonded NdFeB Permanent Magnet, IEEE Trans. Magn. 50 (2014) 697-700.

[9] O. Gutfleisch, K. Güth, T.G. Woodcock, L. Schultz, Recycling Used Nd-Fe-B Sintered Magnets via a Hydrogen-Based Route to Produce Anisotropic, Resin Bonded Magnets, Adv. Energy Mater. 3 (2013) 151155.

[10] P.A. Oyanguren, R.J.J. Williams, Cure of epoxy novolacs with aromatic diamines. I. Vitrification, gelation, and reaction kinetics, J. Appl. Polym. Sci. 47 (1993) 1361-1371.

[11] D.-J. Liaw, B.-Y. Liaw, C.-W. Yu, Synthesis and characterization of new organosoluble polyimides based on flexible diamine, Polymer (Guildf). 42 (2001) 5175-5179. 
[12] A.M. Spring, D. Maeda, M. Ozawa, K. Odoi, F. Qiu, K. Yamamoto, et al., Glass transition temperature control by poly(norbornene-dicarboximide) copolymers, Polym. Bull. 72 (2014) 503-521.

[13] Dupont, Thermal Properties of Elvax ${ }^{\circledR}$ Measured by Differential Scanning Calorimeter (DSC), Elvax Prod. Inf. (2013).

[14] Magnequench, Introducing Anisotropic Powder http://www.mqitechnology.com/downloads/brochures_PDF/MQA-Overview.pdf. (2009).

[15] O. Yousefzade, F. Hemmati, H. Garmabi, M. Mahdavi, Assisted heterogeneous multinucleation and bubble growth in semicrystalline ethylene-vinyl acetate copolymer/expanded graphite nanocomposite foams: Control of morphology and viscoelastic properties, eXPRESS Polym. Lett. 9 (2015) 923.

[16] W. Stark, M. Jaunich, Investigation of Ethylene/Vinyl Acetate Copolymer (EVA) by thermal analysis DSC and DMA, Polym. Test. 30 (2011) 236-242.

[17] D.G. Dikobe, Morphology and properties of polypropylene/ethylene vinyl acetate copolymer/wood powder blend composites, eXPRESS Polym. Lett. 3 (2009) 190-199.

[18] B. John, K.T. Varughese, Z. Oommen, P. Pötschke, S. Thomas, Dynamic mechanical behavior of highdensity polyethylene/ethylene vinyl acetate copolymer blends: The effects of the blend ratio, reactive compatibilization, and dynamic vulcanization, J. Appl. Polym. Sci. 87 (2003) 2083-2099.

[19] H.A. Khonakdar, U. Wagenknecht, S.H. Jafari, R. Hässler, H. Eslami, Dynamic mechanical properties and morphology of polyethylene/ethylene vinyl acetate copolymer blends, Adv. Polym. Technol. 23 (2004) 307315.

[20] R.W. Gao, J.C. Zhang, D.H. Zhang, Y.Y. Dai, X.H. Meng, Z.M. Wang, et al., Dependence of the magnetic properties on the alignment magnetic field for $\mathrm{NdFeB}$ bonded magnets made from anisotropic HDDR powders, J. Magn. Magn. Mater. 191 (1999) 97-100.

[21] R. Gao, J. Zhang, X. Deng, Y. Zhang, Demagnetization process and coercivity for anisotropic HDDR NdFeB bonded magnets, J. Mater. Sci. Technol. 16 (2000) 445-446.

[22] D. Givord, Q. Lu, M.F. Rossignol, P. Tenaud, T. Viadieu, Experimental approach to coercivity analysis in hard magnetic materials, J. Magn. Magn. Mater. 83 (1990) 183-188.

[23] H. Kronmüller, K.-D. Durst, G. Martinek, Angular dependence of the coercive field in sintered Fe77Nd15B8 magnets, J. Magn. Magn. Mater. 69 (1987) 149-157.

[24] G. Varvaro, F. Albertini, E. Agostinelli, F. Casoli, D. Fiorani, S. Laureti, et al., Magnetization reversal mechanism in perpendicular exchange-coupled Fe/L1 0-FePt bilayers, New J. Phys. 14 (2012) 073008.

[25] J.M.D. Coey, K. O’Donnell, New bonded magnet materials (invited), J. Appl. Phys. 81 (1997) 4810. 